\title{
Supporting Information for Publication Fast, Miniaturized, Real-Time Unit for Sampling, Modulation and Separation in Detection of Hazardous Chemicals
}

George C. Pallis* ${ }^{1}$, George P. Psarras ${ }^{1}$, Julia Vamvakari ${ }^{1}$, Matti J. Kuula ${ }^{2}$, Harri Kiljunen ${ }^{2}$, Hanna Hakulinen $^{2}$, Paula Vanninen ${ }^{2}$

${ }^{1}$ T4i engineering Ltd, Advanced Technology Innovation Centre, 5 Oakwood Drive, LE11 3QF, Loughborough, United Kingdom

${ }^{2}$ VERIFIN, Finnish Institute for Verification of the Chemical Weapons Convention, Department of Chemistry, University of Helsinki, P.O. Box 55, FI-00014, Finland

Corresponding Author

* George C. Pallis, PhD

E-mail: g.pallis@t4ieng.com

Phone: +44 (0) 2081442021

Table of Contents

\section{SMS}<smiles>CC(C)OP(C)(=O)F</smiles>
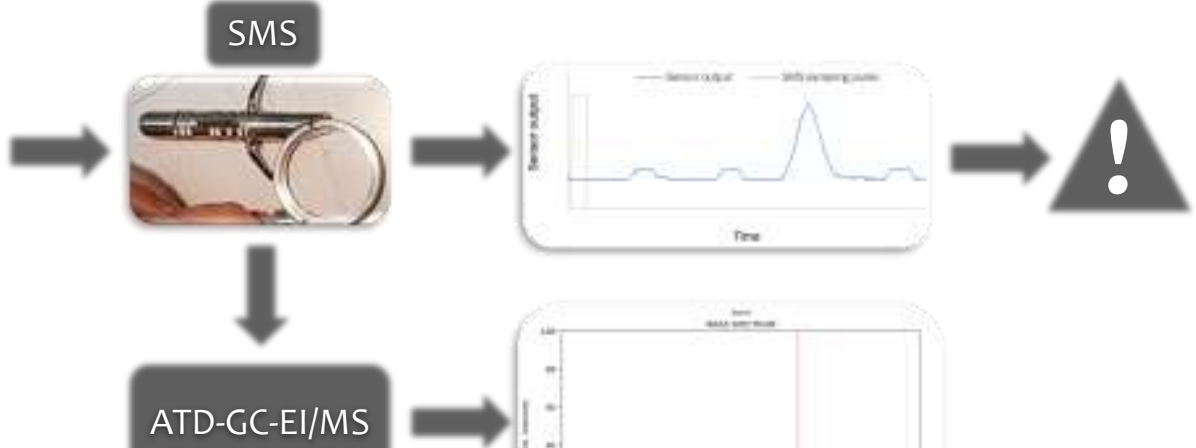

ATD-GC-EI/MS

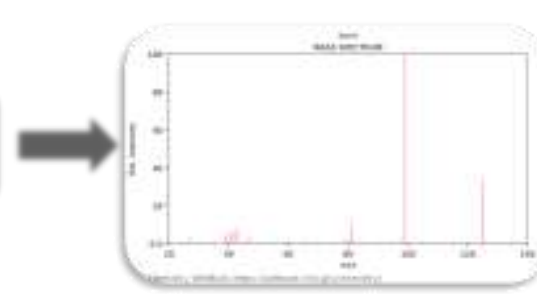


Table S-1. Signal amplitudes and retention times for degradation products of sulphur mustard using SMS fast GC-PID system.

\begin{tabular}{|c|c|c|c|c|c|c|c|c|c|}
\hline \multirow{2}{*}{ Compound } & \multirow{2}{*}{$\begin{array}{c}\text { Concentration } \\
(\mathrm{ppb})\end{array}$} & \multirow{2}{*}{$\begin{array}{c}\text { GC } \\
\text { Temp } \\
\left({ }^{\circ} \mathrm{C}\right)\end{array}$} & \multirow{2}{*}{$\begin{array}{c}\mathrm{GC} \\
\text { Capillary } \\
\text { flow rate } \\
(\mathrm{mL} / \mathrm{min})\end{array}$} & \multicolumn{3}{|c|}{$\begin{array}{l}\text { Signal amplitude when } \\
\text { sampling for } 4 \mathrm{~s}\end{array}$} & \multicolumn{3}{|c|}{ Retention time when sampling for $4 \mathrm{~s}$} \\
\hline & & & & $\begin{array}{l}\text { Mean } \\
(\mathrm{mV})\end{array}$ & Std dev. & RSD $\%$ & Mean (s) & Std dev. & RSD $\%$ \\
\hline Oxathiane & 2500 & \multirow{2}{*}{50} & \multirow{2}{*}{3} & 36 & 0 & $0 \%$ & 22 & 0.3 & $1 \%$ \\
\hline Divinyl Sulfoxide & 2500 & & & 12 & 0.6 & $5 \%$ & 42 & 0.2 & $0 \%$ \\
\hline Oxathiane & 5000 & \multirow{2}{*}{50} & \multirow{2}{*}{3} & 73 & 4.5 & $6 \%$ & 23 & 0.3 & $1 \%$ \\
\hline Divinyl Sulfoxide & 5000 & & & 24 & 3.5 & $15 \%$ & 44 & 0.4 & $1 \%$ \\
\hline Oxathiane & 10000 & \multirow{2}{*}{50} & \multirow{2}{*}{3} & 89 & 3.2 & $4 \%$ & 22 & 0.4 & $2 \%$ \\
\hline Divinyl Sulfoxide & 10000 & & & 30 & 0 & $0 \%$ & 43 & 0.3 & $1 \%$ \\
\hline Oxathiane & 10000 & \multirow{2}{*}{50} & \multirow{2}{*}{3} & 192 & 3.5 & $2 \%$ & 22 & 0.3 & $1 \%$ \\
\hline Divinyl Sulfoxide & 10000 & & & 66 & 3.2 & $5 \%$ & 44 & 0.6 & $1 \%$ \\
\hline Oxathiane & 5000 & \multirow{2}{*}{60} & \multirow{2}{*}{3} & 108 & 5.8 & $5 \%$ & 17 & 0.3 & $2 \%$ \\
\hline Divinyl Sulfoxide & 5000 & & & 48 & 1.7 & $4 \%$ & 30 & 0.1 & $0 \%$ \\
\hline Oxathiane & 10000 & \multirow{2}{*}{60} & \multirow{2}{*}{3} & 192 & 7.6 & $4 \%$ & 17 & 0.2 & $1 \%$ \\
\hline Divinyl Sulfoxide & 10000 & & & 74 & 4.7 & $6 \%$ & 30 & 0.3 & $1 \%$ \\
\hline Oxathiane & 10000 & \multirow{2}{*}{70} & \multirow{2}{*}{3} & 102 & 2.5 & $2 \%$ & 13 & 0.3 & $2 \%$ \\
\hline Divinyl Sulfoxide & 10000 & & & 55 & 4 & $7 \%$ & 23 & 0.2 & $1 \%$ \\
\hline Oxathiane & 5000 & \multirow{2}{*}{90} & \multirow{2}{*}{3} & 111 & 1.2 & $1 \%$ & 10 & 0.2 & $2 \%$ \\
\hline Divinyl Sulfoxide & 5000 & & & 81 & 2.1 & $3 \%$ & 15 & 0.2 & $1 \%$ \\
\hline Oxathiane & 5000 & \multirow{2}{*}{90} & \multirow{2}{*}{3} & 34 & 40.4 & $120 \%$ & 9 & 0.7 & $8 \%$ \\
\hline Divinyl Sulfoxide & 5000 & & & 41 & 30.7 & $74 \%$ & 14 & 0.4 & $3 \%$ \\
\hline
\end{tabular}

